\title{
Quantum-relativistic hydrodynamic model for a spin-polarized electron gas interacting with light
}

\author{
Omar Morandi, Jens Zamanian, " Giovanni Manfredi, and Paul-Antoine Hervieux \\ Institut de Physique et Chimie des Matériaux de Strasbourg and Labex NIE, Université de Strasbourg, CNRS UMR 7504 BP 43, \\ F-67034 Strasbourg Cedex 2, France
}

(Received 10 February 2014; published 18 July 2014)

\begin{abstract}
We develop a semirelativistic quantum fluid theory based on the expansion of the Dirac Hamiltonian to second order in $1 / c$. By making use of the Madelung representation of the wave function, we derive a set of hydrodynamic equations that comprises a continuity equation, an Euler equation for the mean velocity, and an evolution equation for the electron spin density. This hydrodynamic model is then applied to study the dynamics of a dense and weakly relativistic electron plasma. In particular, we investigate the impact of the quantum-relativistic spin effects on the Faraday rotation in a one-dimensional plasma slab irradiated by an x-ray laser source.
\end{abstract}

DOI: 10.1103/PhysRevE.90.013103

PACS number(s): 52.38.-r, 31.15.aj, 03.65.Pm

\section{INTRODUCTION}

During the past decade, an increasing effort has been devoted to the theoretical and experimental study of laser-matter interactions [1-4]. The experiments are usually performed by irradiating a solid target with a high-intensity laser pulse, which can vaporize the target and create a dense plasma, where the electrons may sometimes be accelerated to relativistic velocities. In some high-density plasmas, quantum effects have also been observed [5].

On the other hand, laser-matter experiments are routinely conducted in condensed-matter and nanophysics [6-8]. Typically, a nanometric system (thin metal film, nanoparticle) is excited with an intense and ultrashort (femto- or attosecond) laser pulse, and the charge and spin dynamics is probed by measuring the change of the transmitted or reflected polarization of the light. In such "pump-probe" experiments, the system is first perturbed by a stronger pulse (the pump), followed by a second weaker pulse (the probe) that acts as a diagnostic tool. By modulating the relative amplitude of the signals, as well as the delay between the pump and the probe, it is possible to measure with great precision the relaxation dynamics of the electron gas. Although earlier experiments [6] mainly used visible light, high-intensity microwave and x-ray lasers are now currently used in pump-probe experiments. By varying the laser frequency and intensity, various dynamical regimes can be attained [9-11].

Electromagnetic pulses with intensity of the order of $10^{18} \mathrm{Wcm}^{-2}$ are able to break the internal Coulomb field of the atoms in a solid. Thin films irradiated with such intense sources of microwave radiation can expel electrons with extremely high energies (10-100 MeV) [12,13]. Various experiments have confirmed that bunches of electrons can be expelled from a solid by the electromagnetic field pressure. These electrons are locked on the crest of the electric field of the laser and can follow the electromagnetic field for many cycles, reaching ultrarelativistic velocities [13]. The relativistic Doppler effect was also observed in experiments on nanometric thin foils irradiated with strong laser pulses [14].

In the $\mathrm{x}$-ray domain, lasers with intensity of $10^{14} \mathrm{Wcm}^{-2}$ and energy around $1 \mathrm{keV}$ (corresponding to a wave length

\footnotetext{
*Present address: Department of Physics, Umeå University, SE-901 87 Umeå Sweden.
}

around $1 \mathrm{~nm}$ ) are currently applied to different areas $[15,16]$. Because of the fine spatial resolution that they provide, devices based on such lasers can be used to investigate the local nonequilibrium spin dynamics of an excited system. With this approach, various physical systems were probed, including biological systems [17], metallic films containing magnetic walls [18], and the femtosecond spin response of thin metallic layers [8].

Under such extreme conditions, the numerical simulation of the relativistic electron motion is a primary tool for the interpretation of the experimental results. A widely used approach relies on the application of statistical Monte Carlo methods and particle-in-cell (PIC) simulations [19-21]. These approaches are based on the classical relativistic equations of motion and discard spin and other quantum effects. However, various theoretical studies have revealed that the dynamics of a plasma interacting with an intense electromagnetic field may be modified by spin-dependent effects. For instance, Walser and Keitel [22] observed that the spin-orbit interaction generates some out-of-plane acceleration that is not present in the purely classical relativistic theory. Other theoretical studies, based on the analysis of the motion of a Dirac wave packet, showed that the spin precession induced by a laser field is also modified by quantum-mechanical corrections [23-25]. Thus, the frequency and amplitude of the oscillations of the quantum spin precession could differ significantly from the classical evolution of an equivalent magnetic moment.

In this paper, we study the evolution of a semirelativistic electron plasma where quantum-mechanical effects (particularly the spin dynamics) also play a decisive role. Our approach is based on the quantum hydrodynamic (QHD) representation, which governs the evolution of a small number of macroscopic quantities such as the particle density, average velocity, and average polarization (spin). Relativistic corrections are obtained from the Dirac Hamiltonian, developed to second order in the inverse speed of light by means of the Foldy-Wouthuysen transformation. In other areas of research (particularly quantum chemistry and atomic physics), relativistic corrections to the many-electron dynamics are usually taken into account through relativistic density functional theory (DFT) or HartreeFock methods [26-30]. These approaches-although very fundamental and potentially exact-are in general extremely complex to handle either analytically or numerically, and rarely include spin effects. For these reasons, QHD models 
can provide a simpler alternative that is computationally less demanding but still captures the main physical effects.

We apply the QHD equations to the study of traveling electromagnetic waves in a spin-polarized plasma slab. Interestingly, our simulations show that the inclusion of the spin dynamics has a significant impact on the polarization of the light reflected by or transmitted through the plasma. These spin-related effects could in principle be measured using state-of-the art spectroscopy techniques.

The present paper is organized as follows. In Sec. II, the details of the derivation of the hydrodynamic model are given. In Sec. III, the model is applied to the study of a dense and weakly relativistic electron plasma, with particular emphasis on the effects related to the electron spin. Finally, in Sec. IV we draw our conclusions.

\section{DERIVATION OF THE MODEL}

In this section, we derive a quantum hydrodynamical model that describes the electron dynamics, including semirelativistic and quantum effects. The relevant equations of motion are obtained by the semirelativistic expansion of the Dirac Hamiltonian. A well-established method to provide the semirelativistic corrections to the motion was developed by Foldy and Wouthuysen [31,32]. Within this approach, the positive-energy spectrum of the Dirac Hamiltonian (matter) is decoupled from the negative-energy part (antimatter) by means of a suitable quasiunitary transformation. As a result, a hierarchy of asymptotic Hamiltonian operators is obtained, which are expressed in terms of an expansion in the parameter $\beta=v / c$, where $v$ is the typical velocity of the particle and $c$ is the speed of light (equivalently the parameter $1 / \mathrm{m}$, where $m$ is the electron mass, can also be used for the expansion [33]).

With this approach, it is possible to describe the motion of particles that travel at a sizable fraction of the speed of light - a regime that is relatively common in atomic physics, particularly for heavy elements. In condensed-matter systems and dense plasmas, the relativistic regime can be attained when the electrons are accelerated under the action of an intense laser source, an area of research that is rapidly growing [13].

However, it should be mentioned that the FoldyWouthuysen (FW) expansion is not capable of correctly describing the ultrarelativistic regime $(v \sim c)$, and a fortiori the creation of particle-antiparticle pairs. The FW approach can be extended to ultrarelativistic velocities by applying some renormalization techniques [34], but this approach will not be followed here.

Following Foldy and Wouthuysen, the particle motion up to order $\beta^{2}$ is described by the following Hamiltonian

$$
\begin{aligned}
\hat{H}_{\mathrm{FW}}= & \frac{(\hat{\mathbf{p}}-q \mathbf{A})^{2}}{2 m}+q V-\mu_{B} \boldsymbol{\sigma} \cdot \mathbf{B}-\underbrace{\frac{\hbar^{2} q}{8 m^{2} c^{2}} \nabla \cdot \mathbf{E}}_{H_{D}} \\
& -\underbrace{\frac{\hbar q}{4 m^{2} c^{2}} \boldsymbol{\sigma} \cdot \mathbf{E} \times(\hat{\mathbf{p}}-q \mathbf{A})+\frac{\hbar q}{8 m^{2} c^{2}} \boldsymbol{\sigma} \cdot(\hat{\mathbf{p}} \times \mathbf{E})}_{H_{\mathrm{SO}}},
\end{aligned}
$$

where $m$ is the electron rest mass, $q$ is the charge, $\hat{\mathbf{p}}$ is the momentum operator, $\mu_{B}$ is the Bohr magneton, and $\sigma$ is the
Pauli matrix vector. This Hamiltonian models the interaction of an electron with a classical field described by the electric and magnetic fields $\mathbf{E}$ and $\mathbf{B}$, or equivalently by the scalar and vector potentials, $V$ and $\mathbf{A}$. In the present work, we shall adopt a mean-field approach, whereby the electromagnetic fields are obtained self-consistently from the distributions of charges and currents in the system (see Sec. III below). We also note that the FW Hamiltonian Eq. (1) is a $2 \times 2$ matrix of operators, in contrast to the $4 \times 4$ Dirac Hamiltonian. The domain of $\hat{H}_{\mathrm{FW}}$ is the standard Hilbert space of a spin- $1 / 2$ particle and the resulting evolution equation will be of the Pauli type.

In Eq. (1), the first two terms form the standard Schrödinger Hamiltonian in the presence of an electromagnetic field, whereas the third term is the Pauli spin term (Zeeman effect). The next terms constitute the relativistic corrections: the Darwin term $H_{D}$ and the spin-orbit coupling (SOC) $H_{\mathrm{SO}}$. The former can be interpreted as the first correction to the electric potential induced by the relativistic quivering motion of the electron (Zitterbewegung). A detailed description of the FW Hamiltonian containing the explanation of the physical meaning of the various terms of Eq. (1) can be found in Ref. [35].

Here, we are interested in the study of the quantum motion of a relativistic particle-particularly the corrections that are due to the electron spin-in a semiclassical and semirelativistic regime. As is well known, the spin is one of the physical quantities that is mostly affected by the quantum and relativistic nature of the particle. The most celebrated example is the spin-orbit interaction, which affects crucially, for example, the spectrum of a valence electron. The spin-orbit interaction can be classified as a third-order correction in the quantities $\hbar$ and $c^{-1}$ [as can be seen in Eq. (1), the spin-orbit term is proportional to $\left.\hbar c^{-2}\right]$. Consistently with the semirelativistic FW expansion, we shall derive here the QHD equation of motion up to fourth order, retaining terms $O\left(\hbar^{a} c^{-b}\right)$ with $a+b \leqslant 4$. For the sake of simplicity, we will derive explicitly the evolution equation only up to the third order, while the fourth-order terms will be shown without proof. In the numerical simulations of Sec. III, we shall restrict the calculations to third order.

We derive the quantum-mechanical evolution equation for the hydrodynamical variables [particle density $n(\mathbf{x}, t)$, spin density $\mathbf{s}(\mathbf{x}, t)$, and mean velocity $\mathbf{u}(\mathbf{x}, t)]$ by applying the Madelung transformation [36]. This approach dates back to 1926 when E. Madelung realized that, in the case of a spinless particle, the Schrödinger equation can be put in the following hydrodynamic form:

$$
\begin{aligned}
\frac{\partial n}{\partial t}+\nabla \cdot(n \mathbf{u}) & =0, \\
\frac{\partial \mathbf{u}}{\partial t}+\mathbf{u} \cdot \nabla \mathbf{u}+\frac{q \nabla V}{m}-\frac{\hbar^{2}}{2 m^{2}} \nabla\left(\frac{\Delta \sqrt{n}}{\sqrt{n}}\right) & =0,
\end{aligned}
$$

where the density $n$ and the velocity $\mathbf{u}$ are related to the wave function $\psi$ by $\psi=\sqrt{n} e^{i S / \hbar}$ and $\mathbf{u}=m^{-1} \nabla S$. The QHD equations have the form of an irrotational, compressible, and isothermal Euler system with an additional term, of order $\hbar^{2}$, sometimes referred to as the Bohm potential or quantum pressure [37]. As an alternative to the Madelung transformation, 
QHD models can be derived from a quantum kinetic approach based on the Wigner quasiprobability function [38].

In order to extend the Madelung approach to spin- $1 / 2$ particles, one can generalize the Madelung ansatz as follows [39]:

$$
\psi(\mathbf{x}, t)=\sqrt{n(\mathbf{x}, t)} e^{i S(\mathbf{x}, t) / \hbar} \phi(\mathbf{x}, t),
$$

where the phase $S$ is a real function, and $\phi$ is a normalized twocomponent spinor $\left(\phi^{\dagger} \phi=1\right)$. The spin probability density can be constructed as $\mathbf{S}=\phi^{\dagger} \boldsymbol{\sigma} \phi$. In the case of a particle with spin, it is more difficult to define an average velocity. A possible strategy is to first compute the time derivative of the density $n$ and then impose that the continuity equation be satisfied. It is convenient to write the particle velocity as the sum of two terms: $\mathbf{u} \equiv \mathbf{v}+\boldsymbol{w}$, where $\mathbf{v}$ and $\boldsymbol{w}$ are, respectively, the fluid and spin velocities

$$
\begin{aligned}
\mathbf{v} & \equiv \frac{1}{m} \nabla S-\frac{q}{m} \mathbf{A}-\frac{i \hbar}{m} \phi^{\dagger} \nabla \phi, \\
\boldsymbol{w} & \equiv-\frac{q \hbar}{4 m^{2} c^{2}} \mathbf{S} \times \mathbf{E} .
\end{aligned}
$$

By using the Schrödinger equation with the FW Hamiltonian $i \hbar \partial_{t} \psi=\hat{H}_{\mathrm{FW}} \psi$, it is easy to verify that the continuity equation holds true:

$$
\partial_{t} n+\nabla \cdot(n \mathbf{u})=0 .
$$

We see that in our formulation the velocity field $\mathbf{u}$ has two spin-dependent contributions. The first enters the fluid velocity $\mathbf{v}$ and was already present in the nonrelativistic theory [39,40], while the second $(\boldsymbol{w})$ is a relativistic correction that also appears in the semirelativistic kinetic theory [41,42].

We now derive the evolution equation for the spin density $\mathbf{s}=\phi^{\dagger} \boldsymbol{\sigma} \phi$. We obtain

$$
\partial_{t} \mathbf{s}=\frac{\mathbf{s}}{n} \nabla \cdot(n \mathbf{u})+\frac{2}{n} \operatorname{Re}\left\{\psi^{\dagger} \boldsymbol{\sigma} \partial_{t} \psi\right\},
$$

where we used the continuity Eq. (6). The time derivative of the particle wave function was obtained by calculating the matrix elements of the FW Hamiltonian in the Madelung representation (details are given in Appendix A). By using Eqs. (A15)-(A19), we get

$$
\begin{aligned}
\partial_{t} \mathbf{s}= & \frac{\mathbf{s}}{n}(\boldsymbol{w} \cdot \nabla n)+\mathbf{s}(\nabla \cdot \mathbf{u})+\frac{q}{m} \mathbf{s} \times \mathbf{B} \\
& +\frac{\hbar}{2 m n} \partial_{j}\left(n \mathbf{s} \times \partial_{j} \mathbf{s}\right)+\frac{q}{m^{2} c^{2}} \\
& \times\left[\frac{\hbar}{n}(\mathbf{E} \times \nabla n) \times \mathbf{s}+2 m(\mathbf{E} \times \mathbf{v})+\hbar \mathbf{b}-\hbar \nabla \times \mathbf{E}\right],
\end{aligned}
$$

where the components of the vector $\mathbf{b}$ are defined as

$$
b_{i} \equiv \epsilon_{i r l} \epsilon_{r j k} E_{j} \partial_{k} s_{l} \text {. }
$$

Here, $\epsilon_{i r l}$ is the Levi-Civita symbol and we use the Einstein summation convention on repeated indices, which run on the three spatial coordinates $(x, y, z)$. It is more convenient to express the temporal evolution of the spin density in Eq. (8) in terms of the convective derivative $D / D t \equiv \partial / \partial_{t}+\mathbf{u} \cdot \nabla$.
After some simple algebra, we obtain

$$
\frac{D \mathbf{s}}{D t}=\mathcal{S}(n, \mathbf{s}, \mathbf{u})
$$

where

$$
\mathcal{S} \equiv \frac{q}{m} \mathbf{S} \times\left(\mathbf{B}-\frac{\mathbf{u} \times \mathbf{E}}{2 c^{2}}+\mathbf{B}^{*}\right)+\frac{q \hbar}{2 m^{2} c^{2}}[(\mathbf{E} \times \mathbf{s}) \cdot \nabla] \mathbf{s}
$$

and

$$
\mathbf{B}^{*} \equiv \frac{\hbar}{2 q n}\left(\partial_{j} n \partial_{j} \mathbf{s}\right)+\frac{q \hbar}{4 m^{2} c^{2} n} \mathbf{s} \times[\nabla \times(n \mathbf{E})] .
$$

Various corrective terms to the classical precessional motion appear in Eqs. (9)-(11). The first term describes the spin precession in the presence of the Lorentz-transformed magnetic field $\mathbf{B}-\mathbf{u} \times \mathbf{E} /\left(2 c^{2}\right)$, with the additional contribution of the effective field $\mathbf{B}^{*}$. This effective field is a purely quantum mechanical effect that causes the spin to precess even in the absence of any external field [40].

We now consider the evolution equation for the velocity field. From Eq. (3), we have

$$
\partial_{t} S+i \hbar \phi^{\dagger} \partial_{t} \phi=\frac{1}{n} \operatorname{Im}\left\{\hbar \psi^{\dagger} \partial_{t} \psi\right\} .
$$

Taking the derivative of Eq. (12) with respect to the $i$ th component, we obtain

$$
\begin{aligned}
\partial_{i} & {\left[\partial_{t} S+i \hbar\left(\phi^{\dagger} \partial_{t} \phi\right)\right] } \\
& =\partial_{t}\left[\partial_{i} S+i \hbar\left(\phi^{\dagger} \partial_{i} \phi\right)\right]-\frac{\hbar}{2}\left(\mathbf{s} \times \partial_{i} \mathbf{s}\right) \cdot\left(\partial_{t} \mathbf{s}\right),
\end{aligned}
$$

where we used Eq. (A3) in Appendix A. Equations (4), (12), and (13) lead to

$$
\begin{aligned}
\partial_{t} v_{i}= & \frac{\hbar}{2 m}\left(\mathbf{s} \times \partial_{i} \mathbf{s}\right) \cdot\left(\partial_{t} \mathbf{s}\right)-\frac{q}{m} \partial_{t} A_{i} \\
& +\frac{1}{m} \partial_{i}\left(\frac{1}{n} \operatorname{Im}\left\{\hbar \psi^{\dagger} \partial_{t} \psi\right\}\right) .
\end{aligned}
$$

By using Eqs. (A10)-(A14) in Appendix A, we obtain

$$
\begin{aligned}
\partial_{t} v_{i}+u_{j} \partial_{i} v_{j}= & \frac{\hbar}{2 m}\left(\mathbf{s} \times \partial_{i} \mathbf{s}\right) \cdot\left(\partial_{t} \mathbf{s}\right)-\frac{q}{m} \partial_{t} A_{i}-v_{j} \partial_{i} w_{j} \\
& +\partial_{i}\left[\frac{\hbar^{2}}{2 m^{2}} \frac{\nabla^{2} \sqrt{n}}{\sqrt{n}}-\frac{\hbar^{2}}{8 m^{2}}\left(\partial_{k} s_{j}\right)^{2}\right. \\
& \left.-\frac{q}{m} V+\frac{\mu_{B}}{m} \mathbf{s} \cdot \mathbf{B}\right] .
\end{aligned}
$$

In order to proceed, we note that, for every vector $\mathbf{a}$, we have

$$
\begin{aligned}
\sum_{j} a_{j} \partial_{i} v_{j}= & (\mathbf{a} \cdot \nabla) v_{i}-\frac{q}{m}[\mathbf{a} \times \mathbf{B}]_{i} \\
& -\frac{\hbar}{2 m}\left(\mathbf{s} \times \partial_{i} \mathbf{s}\right) \cdot[(\mathbf{a} \cdot \nabla) \mathbf{s}] .
\end{aligned}
$$

Equation (17) can be obtained by using

$$
\begin{aligned}
\partial_{i} v_{j}= & \partial_{j} v_{i}+\frac{q}{m}\left(\partial_{j} A_{i}-\partial_{i} A_{j}\right) \\
& +\frac{i \hbar}{m}\left[\partial_{j}\left(\phi^{\dagger} \partial_{i} \phi\right)-\partial_{i}\left(\phi^{\dagger} \partial_{j} \phi\right)\right],
\end{aligned}
$$


which follows directly from the definition of the velocity $\mathbf{v}$ in Eq. (4) and from Eq. (A3). Using the above results, we obtain

$$
\begin{aligned}
\frac{D v_{i}}{D t}= & \frac{q}{m}[\mathbf{E}+\mathbf{v} \times \mathbf{B}]_{i}+\frac{\hbar}{2 m}\left(\mathbf{s} \times \partial_{i} \mathbf{s}\right) \cdot \frac{D \mathbf{s}}{D t}-v_{j} \partial_{i} w_{j} \\
& +\partial_{i}\left[\frac{\hbar^{2}}{2 m^{2}} \frac{\nabla^{2} \sqrt{n}}{\sqrt{n}}-\frac{\hbar^{2}}{8 m^{2}}\left(\partial_{k} s_{j}\right)^{2}+\frac{\mu_{B}}{m} \mathbf{s} \cdot \mathbf{B}\right]
\end{aligned}
$$

where the total spin derivative is given by Eq. (9). After some straightforward calculations, we obtain the evolution equation of the total velocity field $\mathbf{u}$ :

$$
\frac{D \mathbf{u}}{D t}=\mathcal{U}(n, \mathbf{s}, \mathbf{u}),
$$

where

$$
\begin{aligned}
\mathcal{U} \equiv & \frac{q}{m}(\mathbf{E}+\mathbf{u} \times \mathbf{B})+\frac{\hbar^{2}}{2 m^{2}} \nabla\left(\frac{\Delta \sqrt{n}}{\sqrt{n}}\right)+\frac{q \hbar}{2 m^{2}} s_{j} \nabla\left[B_{j}-\frac{1}{2 c^{2}}(\mathbf{u} \times \mathbf{E})_{j}\right]-\frac{\hbar^{2}}{4 m^{2} n} \partial_{k}\left[n\left(\nabla s_{j}\right)\left(\partial_{k} s_{j}\right)\right] \\
& -\frac{q \hbar}{4 m^{2} c^{2}} \mathbf{s} \times\left(\partial_{t}+\mathbf{u} \cdot \nabla\right) \mathbf{E}-\frac{q \hbar}{4 m^{2} c^{2}}[(\mathbf{s} \times \mathbf{E}) \cdot \nabla] \mathbf{u}-\frac{q^{2} \hbar}{4 m^{3} c^{2}}[\mathbf{s} \times(\mathbf{E} \times \mathbf{B})] .
\end{aligned}
$$

The evolution Eq. (20) shows that, in the semiclassical and semirelativistic regime, the motion is strongly affected by the interaction between the spin and the velocity of the particle. When the spin is neglected, we obtain the simple Madelung system of Eqs. (2). In the general case, the evolution equation takes a complex form with an intricate interplay between spin and velocity degrees of freedom. In particular, the term $\frac{q \hbar}{2 m^{2}} s_{j} \nabla\left[B_{j}-\frac{1}{2 c^{2}}(\mathbf{u} \times \mathbf{E})_{j}\right]$ describes the deviation of the particle velocity caused by the presence of an inhomogeneous Lorentz-transformed magnetic field (this is the effect responsible for the spin-dependent deviation of an electron beam in a typical Stern-Gerlach experiment). Further, the term $\frac{\hbar^{2}}{4 m^{2} n} \partial_{k}\left[n\left(\nabla s_{j}\right)\left(\partial_{k} s_{j}\right)\right]$ is known as the spin potential and is described in some details in Ref. [40].

As mentioned above, the calculations leading to the evolution Eq. (20) are correct up to the third order in the small parameters $\hbar$ and $c^{-1}$. For the sake of completeness, we provide here also the result at the next (fourth) order, i.e., $\hbar^{a} c^{-b}$, with $a+b \leqslant 4$ :

$$
\begin{aligned}
\frac{D u_{i}}{D t}= & \mathcal{U}_{i}-\frac{q \hbar^{2}}{8 m^{3} c^{2}} \partial_{i}(\nabla \cdot \mathbf{E})-\frac{q \hbar^{2}}{8 m^{3} c^{2} n} \\
& \times\left\{\epsilon_{i j k} E_{k} \partial_{n}\left(n \epsilon_{j l m} s_{l} \partial_{n} s_{m}\right)-\epsilon_{j k l} s_{k}\left(\partial_{i} s_{l}\right)[\nabla \times(n \mathbf{E})]_{j}\right\} \\
& +\frac{q \hbar^{2}}{8 m^{3} c^{2}}\left[\epsilon_{o j k} \epsilon_{n l m} s_{l} s_{j} E_{k}\left(\partial_{i} s_{m}\right)\left(\partial_{o} s_{n}\right)\right. \\
& \left.-\epsilon_{o j k} \epsilon_{o l m} \partial_{i}\left(E_{j} s_{l} \partial_{k} s_{m}\right)\right] .
\end{aligned}
$$

We will not include the fourth-order terms in the final version of our model that will be solved numerically in Sec. III.

Equations (6), (9), and (20) constitute the model that we shall use in the next section as the basis of our numerical simulations of an intense laser pulse impinging on a solid target. Similar relativistic equations were obtained in the past directly from the Dirac equation [43,44]. In particular, the work of Asenjo et al. [43] provides a set of fully covariant hydrodynamic equations that is consistent with the nonrelativistic theory [39] in the limit of low velocities. The covariant model [43] is nevertheless very complex to implement in practical situations, notably for numerical applications. Our semirelativistic fluid model-based on an extension of Pauli's two-component spinor approach in contrast to the fourcomponent approach of Ref. [43] - is more easily amenable to numerical studies, as will be shown in the next section.

\section{APPLICATION TO RELATIVISTIC LASER-PLASMA INTERACTIONS}

In this section, we apply the semirelativistic QHD model to study the propagation of an electromagnetic wave (referred to as the "light") in a plasma slab. We will focus on the effects induced by the spin-dependent semirelativistic corrections on the polarization of the incoming light. We consider a simplified slab geometry where the plasma is finite along one dimension ( $z$ direction), whereas it is uniform in the transverse plane. Such plasmas can be created by irradiating a solid film target with an intense laser pulse that extracts the electrons from the solid [2,12], while the more massive ions are still immobile and can be modeled by a continuous positive charge density $n_{0}$ (jellium model). Although the model is essentially one-dimensional (1D), the spin direction is maintained as a three-dimensional vector $\mathbf{s}(z, t)$. Assuming that the dynamical variables $n, \mathbf{u}$, and $\mathbf{s}$ depend only on the longitudinal coordinate $z$, the system of Eqs. (6), (9), and (20) simplifies to

$$
\partial_{t} n+\partial_{z}\left(u_{z} n\right)=0
$$

$$
\begin{aligned}
\partial_{t} \mathbf{s}+ & \left(u_{z}-v_{c}\right) \partial_{z} \mathbf{s} \\
=\mathbf{s} \times & {\left[\beta \xi \mathbf{B}-\frac{\xi \beta^{2}}{2} \mathbf{u} \times \mathbf{E}+\frac{\gamma}{2 n} \partial_{z}\left(n \partial_{z} \mathbf{s}\right)+\mathbf{s} \times \mathbf{R}\right], } \\
\partial_{t} \mathbf{u}+\left(u_{z}+\frac{v_{c}}{2}\right) \partial_{z} \mathbf{u} & \xi(\mathbf{E}+\beta \mathbf{u} \times \mathbf{B})+\frac{\gamma}{2}\left[\xi \beta\left(\mathbf{s} \cdot \partial_{z} \mathbf{B}\right)\right. \\
= & \\
& \left.-\frac{\gamma}{2 n} \partial_{z}\left(n\left|\partial_{z} \mathbf{s}\right|^{2}\right)-\frac{\gamma \beta^{2} \xi}{4} \partial_{z}^{2} E_{z}\right] \hat{\mathbf{e}}_{z} \\
& -\frac{\gamma \xi \beta^{2}}{4}\left[\xi \mathbf{s} \times(\mathbf{E} \times \mathbf{B})+\mathbf{s} \times\left(\partial_{t}+u_{z} \partial_{z}\right) \mathbf{E}\right]
\end{aligned}
$$

where $\hat{\mathbf{e}}_{z}$ is the unit vector along the $z$ axis and we defined

$$
\begin{aligned}
& v_{c}=\frac{\gamma \xi \beta^{2}}{2}(\mathbf{E} \times \mathbf{s})_{z}, \\
& \mathbf{R}=\frac{\gamma \xi \beta^{2}}{4 n} \nabla \times(n \mathbf{E}) .
\end{aligned}
$$

All the quantities that appear in Eqs. (23)-(25) are dimensionless. We applied the following scaling: $z^{\prime}=z / l_{0}, t^{\prime}=t / t_{0}$, $n^{\prime}=n l_{0}^{3}, \mathbf{u}^{\prime}=\mathbf{u} t_{0} / l_{0}, \mathbf{E}^{\prime}=\mathbf{E} / E_{0}, \mathbf{B}^{\prime}=\mathbf{B} E_{0} / c$, where the 
primed variables are dimensionless quantities (in order to simplify the notation, we dropped the prime symbol in the final equations). Here, $l_{0}$ and $t_{0}$ are the characteristic length and time of the system, and $E_{0}$ is a typical value of the electric field. With this normalization, the evolution Eqs. (23)-(25) are expressed in terms of the three dimensionless parameters

$$
\gamma=\frac{\hbar t_{0}}{m l_{0}^{2}}, \quad \xi=\frac{q t_{0}^{2} E_{0}}{m l_{0}}, \quad \beta=\frac{l_{0}}{c t_{0}},
$$

where $\alpha=e^{2} /\left(4 \pi \varepsilon_{0} \hbar c\right)$ is the fine-structure constant.

The electric and magnetic fields consist of external and self-consistent fields, the latter being generated by the plasma internal charge density and current. The external fields are the electromagnetic field of the laser (described by the vector potential $\mathbf{A}_{\text {las }}$ ) and an external constant magnetic field $\mathbf{B}_{0}$. We decompose the electric field in the longitudinal and transverse components, $\mathbf{E}=E_{z} \hat{\mathbf{e}}_{z}+\mathbf{E}_{\perp}$. The longitudinal field $E_{z}$ is obtained from Poisson's equation

$$
\partial_{z} E_{z}=\chi\left(n-n_{0}\right),
$$

where $\chi=q /\left(\varepsilon_{0} E_{0} l_{0}^{2}\right)$. The transvere field is given by $\mathbf{E}_{\perp}=$ $-\partial_{t}\left(\mathbf{A}_{\perp}+\mathbf{A}_{\text {las }}\right)$, where the vector potential $\mathbf{A}_{\perp}$ satisfies the d'Alembert equation

$$
\partial_{t}^{2} \mathbf{A}_{\perp}-\beta^{-2} \partial_{z}^{2} \mathbf{A}_{\perp}=\chi \mathbf{j} .
$$

The expression for the current $\mathbf{j}$ is more difficult to determine. In a relativistic framework, the quantum-mechanical current contains other terms beyond the usual Schrödinger expression [35]. The first of such terms is the so-called spin current, which is proportional to the curl of the magnetization density [45]. For the sake of simplicity, we will only retain this correction and write the total current as

$$
\mathbf{j}=n\left(\mathbf{u}+\frac{\gamma}{2} \nabla \times \mathbf{s}\right) .
$$

Finally, the total magnetic field is given by $\mathbf{B}=\mathbf{B}_{\mathrm{elm}}+\mathbf{B}_{0}$ where $\mathbf{B}_{\text {elm }}=\beta^{-1} \nabla \times\left(\mathbf{A}_{\perp}+\mathbf{A}_{\text {las }}\right)$.

We study the evolution of a laser beam propagating along the $z$ direction and impinging on the plasma slab with positive momentum. At $t=0$ the system is at rest and $n(z, t=0)=n_{0}(z)$. The density profile is depicted in Fig. 1. In our simulations, we consider a plasma with a thickness $l_{0}=40 \mathrm{~nm}$ and we study two different regimes of

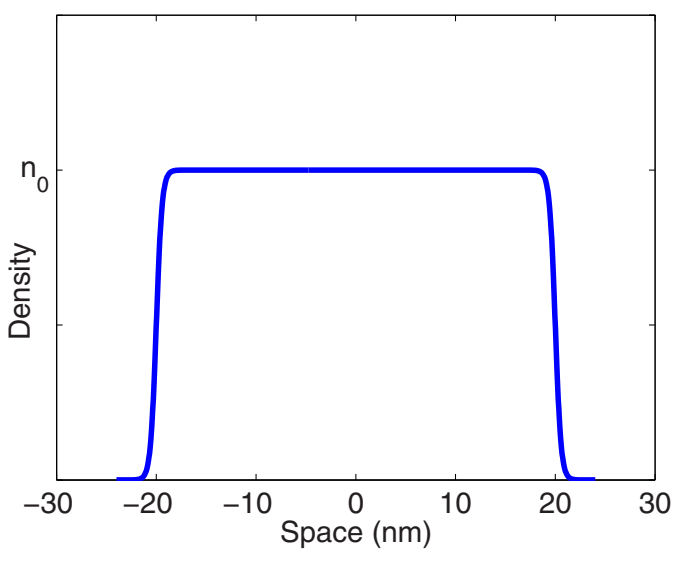

FIG. 1. (Color online) Plot of the ion density profile.
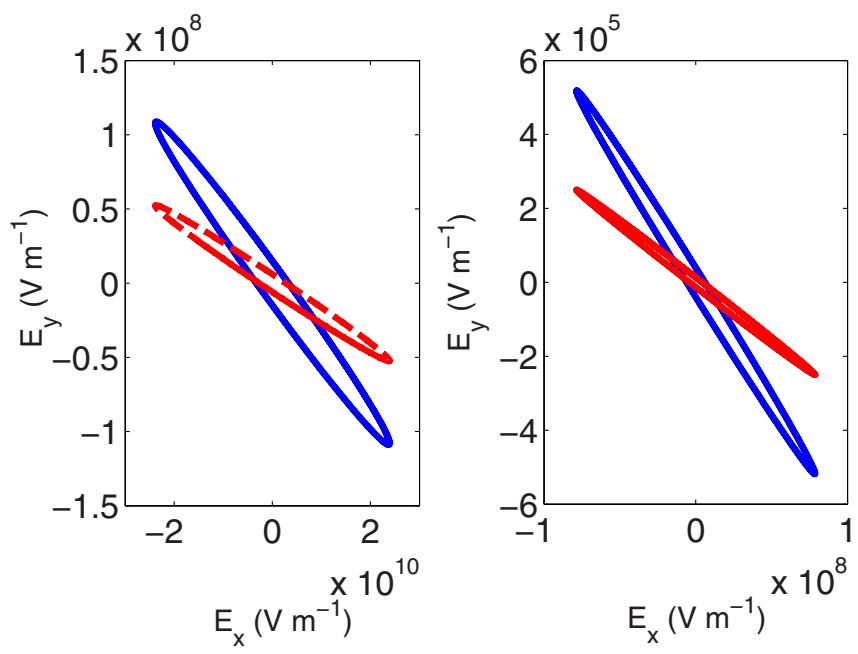

FIG. 2. (Color online) Trajectory of the electric field in the transverse $(x, y)$ plane, for the transmitted (left panel) and reflected (right panel) light, with spin effects (blue continuous line) and without spin effects (red dashed line). The plasma density is $n_{0}=1.5 \times 10^{-1} \mathrm{~nm}^{-3}$ and the laser frequency is equal to $2 \mathrm{keV}$ (wavelength $\lambda=2 \mathrm{~nm}$ ).

plasma density: $n_{0}=1.5 \times 10^{-4} \mathrm{~nm}^{-3}$ (rarefied plasma) and $n_{0}=0.15 \mathrm{~nm}^{-3}$ (dense plasma). The external magnetic field $\mathbf{B}_{0}=10^{-2} \mathrm{~T}$ is uniform and directed along the longitudinal direction $z$. We also assume that the electron gas is initially fully polarized along the same direction; i.e., $\mathbf{s}(0)=\hat{\mathbf{e}}_{z}$. We excite the system by an intense source of linearly polarized light in the $\mathrm{x}$-ray range (laser power $10^{16} \mathrm{~W} / \mathrm{cm}^{2}$ ). The electric field generated by the laser is of the order of $10^{10} \mathrm{~V} / \mathrm{m}$. In the simulations, the laser wavelength $\lambda$ will be varied in the range between $\lambda=l_{0} / 35$ and $\lambda=l_{0}$. The above parameters are compatible with those normally achieved in current laser-solid experiments $[2,12]$.

In the forthcoming description of the numerical results, we will focus on the polarization of the reflected and transmitted
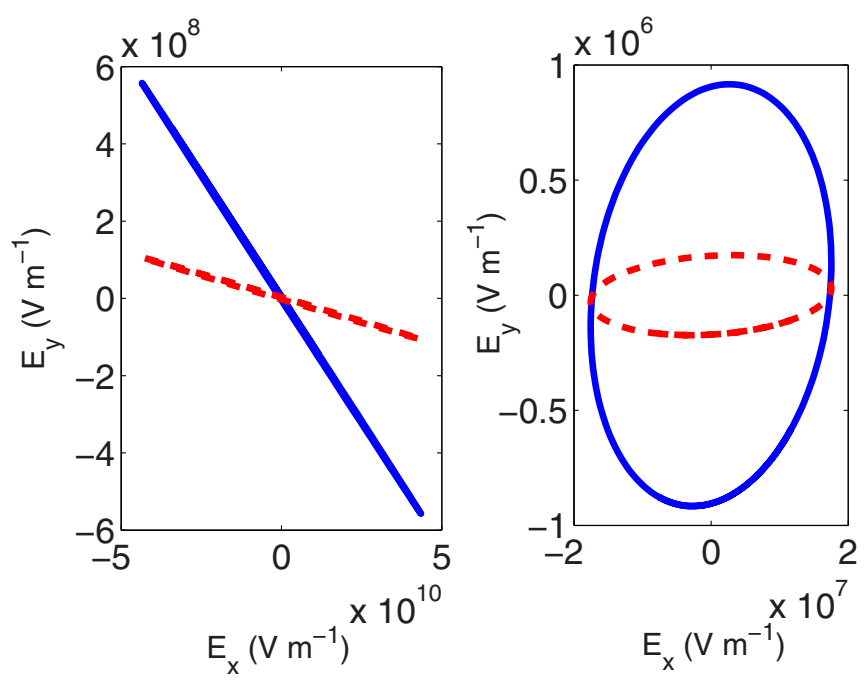

FIG. 3. (Color online) Same as described in the legend of Fig. 2, but for a larger laser frequency equal to $4 \mathrm{keV}$ (wavelength $\lambda=1 \mathrm{~nm})$. 


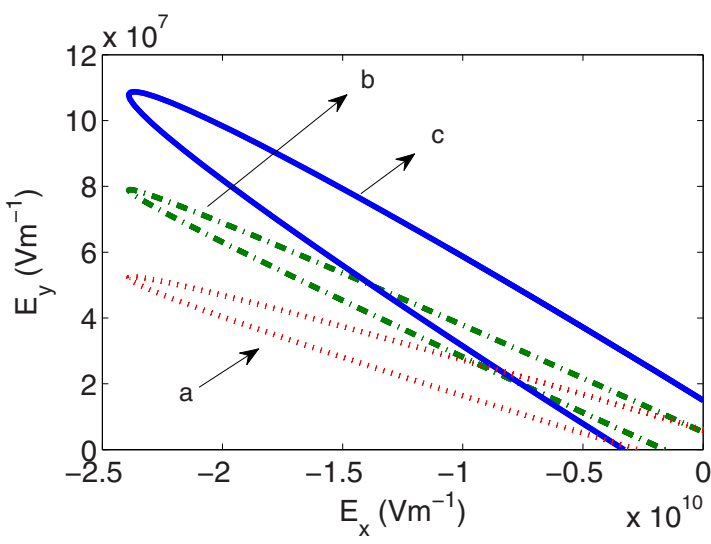

FIG. 4. (Color online) Same as described in the legend of Fig. 2 (left panel) for the transmitted light. (a) Classical case, obtained by setting $\gamma=0$ in Eqs. (23)-(25) and (31); (b) intermediate case with $\gamma=0$ in Eqs. (23) $-(25)$ but $\gamma \neq 0$ in Eq. (31); (c) result from the full model.

light. These quantities are particularly relevant since they could be directly measured in pump-probe experiments. They are related to the Faraday angle for the transmitted light and the Kerr angle for the reflected light $[33,46,47]$, defined as the angle $\theta$ between the components of the electric field in the transverse plane, $\theta=\tan ^{-1}\left(\left|E_{y} / E_{x}\right|\right)$.

The laser pulse is switched on at $t=0$ and, after a delay of nearly 10 periods of the incoming radiation $(T=\lambda / c)$, the system reaches a stationary regime. In Figs. 2 and 3, we show the trajectory of the transverse components of the electric field at the boundaries of the simulation domain, for two values of the laser frequency. For the sake of comparison, we depict (red dashed line) the same quantity for a case where all spin effects are neglected (i.e., by setting $\mathbf{s} \equiv 0$ everywhere). The simulations show that irradiating the plasma with a strong laser pulse can trigger semirelativistic spin effects that have a clear signature in the polarization of the emitted light. In particular, the modification of the Kerr-Faraday angles increases with the laser frequency, as can be seen by comparing Fig. 2 with Fig. 3.

A priori, it is not easy to guess which terms in the evolution Eqs. (23)-(25) have a larger impact on the modification of

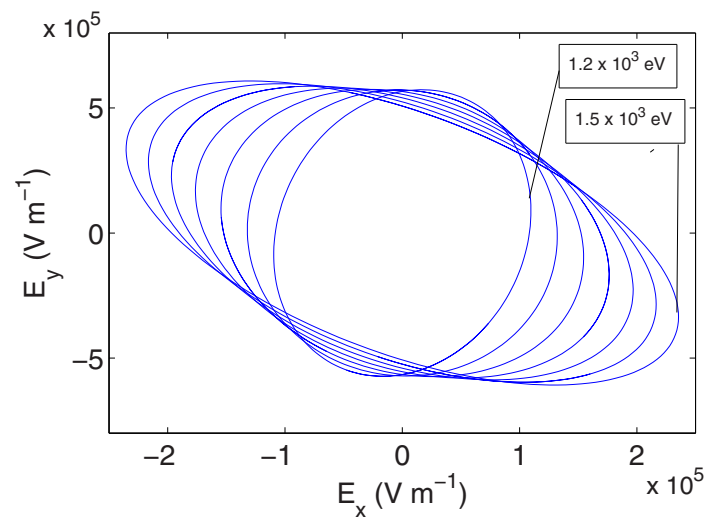

the Kerr-Faraday angles. In order to clarify this point, we performed two further simulations that retain only some of the terms in the equations (see Fig. 4). For the case (a) on the figure, all the quantum terms were suppressed, which amounts to setting $\gamma=0$ everywhere in Eqs. (23)-(25) and also in the definition of the current in Eq. (31) (notice that $\gamma$ is proportional to $\hbar$ ). The run (b) was obtained by setting $\gamma=0$ in Eqs. (23)-(25) but not in Eq. (31); in other words, we took into account the spin-current term, which is a nonrelativistic spin correction. The case (c) represents the result obtained with the full model (including relativistic terms). It is clear from Fig. 4 that, although the nonrelativistic spin-current correction has an impact on the Kerr rotation angle [curve (b)], the relativistic terms also play a significant role. Indeed, the two effects seem to have a comparable importance, at least in the present case.

The study of the variation of the Kerr angle with respect to the laser energy (or frequency) is presented in Fig. 5, where we show the modification of the light polarization for different laser frequencies and two values of the plasma density. The values of the laser frequency are displayed on the figure. The simulations show that, in the dense plasma regime (left panel of Fig. 5), the polarization of the light is strongly influenced by the frequency of the laser.

For low laser frequencies (smaller than $7.5 \times 10^{2} \mathrm{eV}$ ), the behavior of the light polarization is presented in Fig. 6. In the left panel, we depict the ratio between the power of the reflected light and the power injected in the slab by the laser, while in the right panel we show the Kerr angle. The plots show that these quantities display a series of maxima separated by a period of roughly $2 \times 10^{2} \mathrm{eV}$. The origin of these oscillations can be explained in terms of the number of the laser wavelengths contained in the slab thickness, each oscillation corresponding to adding one complete wavelength of the electromagnetic wave inside the slab. The transmission of light through the plasma slab (Fig. 6, left panel) is maximal when the laser wavelength fits the size of the slab. The plot of the Kerr angle (Fig. 6, right panel) displays several peaks that follow a similar pattern.

At the frequency $3 \times 10^{2} \mathrm{eV}$, the electromagnetic wave makes one complete oscillation inside the slab. This is illustrated in Fig. 7, where we depict the mean velocity (right panel) and the self-consistent vector potential A (left panel) at a given time. The density profile is also shown for comparison.

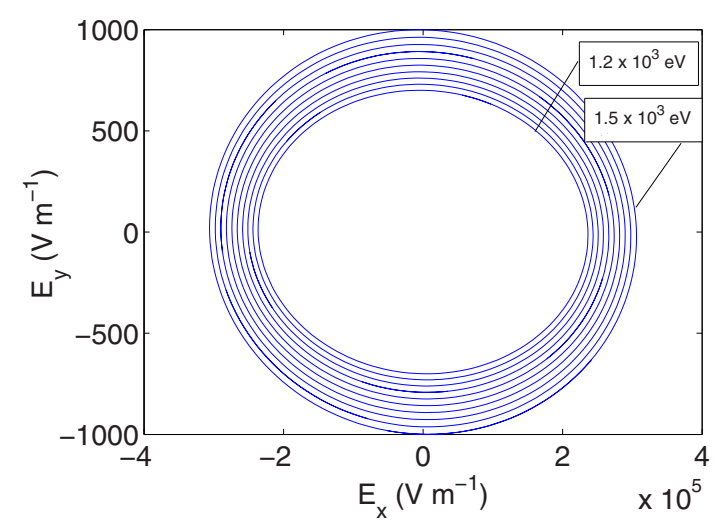

FIG. 5. (Color online) Polarization plane of the reflected light for different laser energies. The curves correspond to energies between 1.2 and $1.5 \mathrm{keV}$, separated by equal intervals of $50 \mathrm{eV}$. Left panel: Plasma density $n_{0}=1.5 \times 10^{-1} \mathrm{~nm}^{-3}$. Right panel: $n_{0}=1.5 \times 10^{-4} \mathrm{~nm} \mathrm{~m}^{-3}$. 

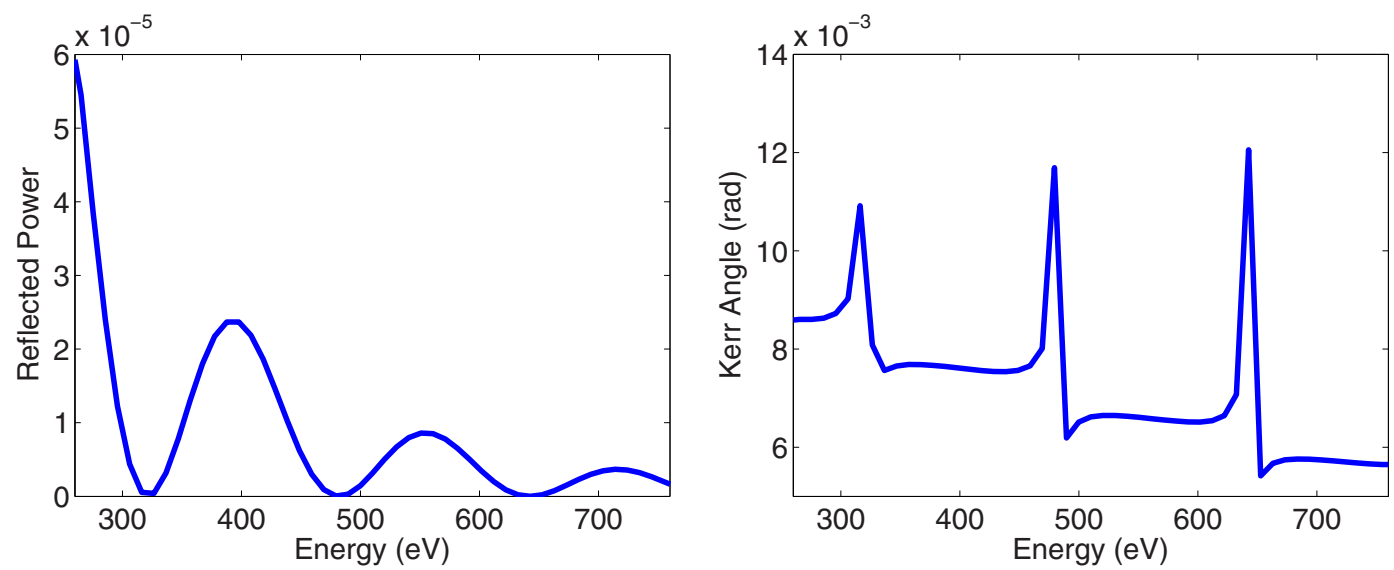

FIG. 6. (Color online) Left panel: Ratio between the power of the reflected light and the laser power as a function of the laser energy. Right panel: Kerr angle as a function of the laser energy.

The right panel of Fig. 7 shows that the laser field can accelerate the plasma up to $0.1 \%$ of the speed of light.

The spin distribution is shown in Fig. 8. We represent the transverse components of the electron spin multiplied by the density profile: $\mathbf{s} n / \max (n)$. This $3 \mathrm{D}$ plot, which displays the spatial and time variation of the spin waves, illustrates one of the principal results of our study of the laser-plasma interaction. It shows that an intense laser wave propagating along the slab excites transverse spin waves with the wavelength of the impinging wave. The spin precession in the transverse plane is mainly produced by the first two terms on the right-hand side of Eq. (24), which are proportional to the total magnetic field and to the plasma current. According to Eq. (31) the plasma current has a contribution proportional to the curl of the spin distribution. For this reason, transverse spin oscillations generate a current and consequently a field directed in the transverse plane. This field is, in general, rotated with respect to the polarization plane of the laser and modifies the Kerr-Faraday angle. Moreover, the spatial gradient of the spin distribution increases with increasing laser frequency, and consequently the modification of the polarization plane of the electromagnetic wave is accentuated, as was shown in Figs. 2 and 3 .
We note that temperature effects were disregarded altogether in our treatment. The reason is that, for the highintensity laser pulses that we considered, the coherent motion is dominant over thermal effects. For instance, for an electron gas at room temperature, the thermal speed is more than 20 times smaller than the coherent velocity gained by the electrons in response to the laser excitation (which is about $0.5 \%$ of the speed of light; see Fig. 7). For longer times, this coherent motion may be converted into heat $[48,49]$, thus leading to thermal effects (e.g., Landau damping) not included in our model. Our results are thus valid for the initial stages of the evolution, during which the coherent motion is dominant. In order to properly include such thermal effects, a kinetic [42], rather than fluid, model should be employed.

\section{CONCLUSION}

Relativistic effects can have an impact on the electron dynamics in heavy atoms, dense plasmas, and condensedmatter systems excited with intense and ultrafast laser pulses. In particular, the electron spin can couple not only to the electric field of the static nuclei (this is the ordinary spin-orbit coupling), but also to the self-consistent mean field generated
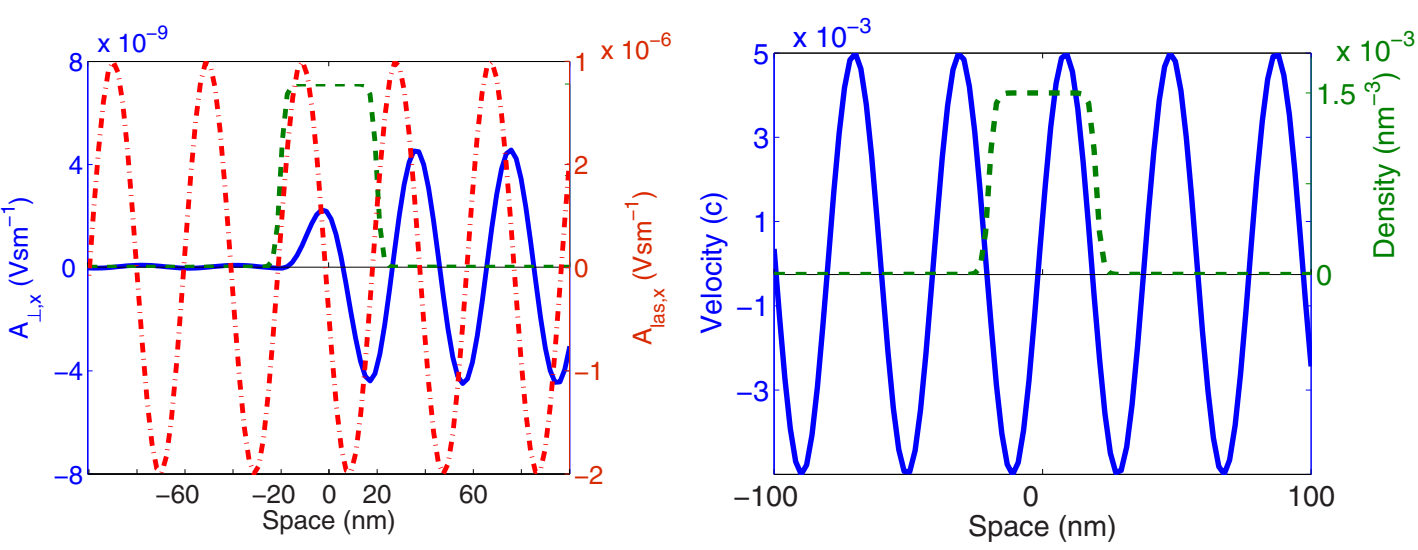

FIG. 7. (Color online) Left panel: Self-consistent vector potential $A_{\perp, x}$ (continuous blue line, left scale) and laser vector potential $A_{\text {las }, x}$ (dash-dotted red line, right scale) along the longitudinal $z$ direction. Note the different scales for the self-consistent and laser potentials. Right panel: Mean plasma velocity (continuous blue line). The dashed green line represents the ion density profile. The laser frequency is $3 \times 10^{2} \mathrm{eV}$, corresponding to a wavelength that fits exactly into the plasma slab. 

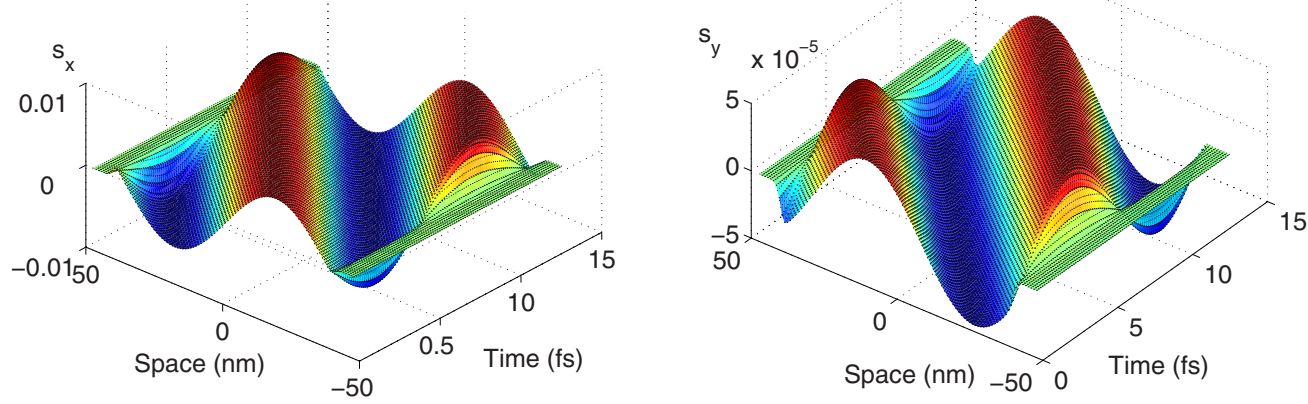

FIG. 8. (Color online) Spin density $n \mathbf{s}(z, t)$ in the transverse plane, as a function of time and the longitudinal coordinate $z$. Left panel: $n s_{x}$. Right panel: $n s_{y}$.

by all the other electrons, or directly to the magnetic and electric fields of the incident laser pulse. In view of this complex variety of possible physical mechanisms, it may be necessary to go beyond the lowest-order description of an electron with spin, i.e., the Pauli equation.

The purpose of this paper was to derive a self-consistent quantum hydrodynamic model that incorporates quantum, spin, and relativistic effects up to second order in $1 / c$. We started from the second-order Hamiltonian derived from the Dirac equation through a Foldy-Wouthuysen transformation. The set of hydrodynamic equations was obtained by performing a Madelung transformation of the wave function. The model comprises a continuity equation, an Euler equation for the mean velocity, and an evolution equation for the spin density.

Using this model, we studied the quantum-relativistic corrections to the electron spin precession in a one-dimensional plasma slab excited by a laser field. The simulations showed that quantum-relativistic effects have a clear signature on the polarization of the laser electromagnetic wave transmitted through the slab. Our results should be relevant to laserplasma interactions at solid-state densities (hence the need to include quantum effects) and for large laser powers (which can accelerate the electrons to weakly relativistic velocities [12]). Other possible areas of applications involve inertial confinement fusion [50] and astrophysical plasmas [51], as well as nanometric systems (nanoparticles, thin films) excited with ultrashort laser pulses in the femto- or attosecond domain [7]. The signature of the spin effects should be detectable using standard spectroscopy techniques.

\section{ACKNOWLEDGMENTS}

We thank the Agence Nationale de la Recherche (project Labex "Nanostructures in Interaction with their Environment") and the European Research Council (Advanced Grant "Atomag," No. ERC-2009-AdG-20090325 247452) for financial support.

\section{APPENDIX A: MATRIX ELEMENTS OF THE FW HAMILTONIAN IN THE MADELUNG REPRESENTATION}

We evaluate the matrix elements of the Foldy-Wouthuysen Hamiltonian for the Madelung hydrodynamic description of the particle wave function. Although the calculations proceed straightforwardly, some technical problems arise concerning the relationship between the microscopic variables $(S, \phi)$ and the macroscopic $(\mathbf{s}, \mathbf{u})$ quantities. We are interested in the following terms:

$$
\begin{gathered}
H_{1} \equiv \frac{1}{n} \operatorname{Re}\left\{\psi^{\dagger} \hat{H} \psi\right\} \\
H_{2} \equiv \frac{1}{n} \operatorname{Im}\left\{\psi^{\dagger} \sigma \hat{H} \psi\right\} .
\end{gathered}
$$

The relationship $\mathbf{s}=\phi^{\dagger} \boldsymbol{\sigma} \phi$ shows that the macroscopic quantity $\mathbf{s}$ is easily obtained from the spinor $\phi$. Equations (A1) and (A2) are nonlinear expressions of the spinor $\phi$. In order to close the equations of motion, Eqs. (24) and (14), $\phi$ should be expressed in terms of $\mathbf{s}$. Although this is, in principle, possible (it is easy to check that the maps are invertible), the direct computation of the inverse map leads to some cumbersome expressions. One way to circumvent this difficulty is to express the product of spinors in some symmetric form that can be easily computed. As a preliminary step, we state here one of the key relationships between the spinor and the spin density, which reads as

$$
\begin{aligned}
I_{3} & \equiv \partial_{j}\left(\phi^{\dagger} \partial_{i} \phi\right)-\partial_{i}\left(\phi^{\dagger} \partial_{j} \phi\right)=-\frac{i}{2}\left(\mathbf{s} \times \partial_{i} \mathbf{s}\right) \cdot\left(\partial_{j} \mathbf{s}\right) \\
i, j & =x, j, z, t .
\end{aligned}
$$

Here, $i, j=x, j, z, t$ are fixed indices where, extending our previous notation, we also include the time variable. The validity of Eq. (A3) can be established with a few calculations that we now detail in the following paragraphs. We have

$$
\begin{aligned}
I_{3}= & \left(\partial_{j} \phi^{\dagger}\right) \partial_{i} \phi-\left(\partial_{i} \phi^{\dagger}\right) \partial_{j} \phi=\partial_{j}\left[\phi^{\dagger}\left(2 \phi \phi^{\dagger}-\sigma \cdot \mathbf{s}\right)\right] \partial_{i} \phi \\
& -\left(\partial_{i} \phi^{\dagger}\right) \partial_{j}\left[\left(2 \phi \phi^{\dagger}-\sigma \cdot \mathbf{s}\right) \phi\right] \\
= & 3 I_{3}-\left(\partial_{j} s_{k}\right) \eta_{k i},
\end{aligned}
$$

where we used Eq. (A5), the relation $\left(\partial \phi^{\dagger}\right) \phi=-\phi^{\dagger} \partial \phi$, and the following general property of the product between two spinors:

$$
\phi \phi^{\dagger}=\frac{1}{2}\left(\sigma_{0}+\mathbf{s} \cdot \boldsymbol{\sigma}\right),
$$

where $\sigma$ denotes the vector of Pauli matrices and $\sigma_{0}$ is the $2 \times 2$ identity matrix. Finally, we define

$$
\eta_{k i} \equiv \phi^{\dagger} \sigma_{k} \partial_{i} \phi-\left(\partial_{i} \phi^{\dagger}\right) \sigma_{k} \phi
$$


By using the identity Eq. (A5), we obtain

$$
\begin{aligned}
\eta_{i j}= & \phi^{\dagger} \sigma_{i} \partial_{j}\left[\left(2 \phi \phi^{\dagger}-\mathbf{s} \cdot \boldsymbol{\sigma}\right) \phi\right] \\
& -\left\{\partial_{j}\left[\phi^{\dagger}\left(2 \phi \phi^{\dagger}-\mathbf{s} \cdot \boldsymbol{\sigma}\right)\right]\right\} \sigma_{i} \phi \\
= & 3 \eta_{i j}-4 s_{i} \phi^{\dagger} \partial_{j} \phi-\left(\partial_{j} s_{k}\right) \phi^{\dagger}\left[\sigma_{i}, \sigma_{k}\right] \phi,
\end{aligned}
$$

where the square brackets denote the commutator. Using the well-known commutation properties of the Pauli matrices $\left[\sigma_{i}, \sigma_{j}\right]=i 2 \varepsilon_{i j k} \sigma_{k}$, one gets

$$
\eta_{i j}=2 s_{i} \phi^{\dagger} \partial_{j} \phi-i \epsilon_{i k l} s_{k} \partial_{j} s_{l} .
$$

Plugging Eq. (A8) in Eq. (A4) yields the following:

$$
2 I_{3}=\left(\partial_{j} \mathbf{s}_{k}\right) \cdot\left[2 \mathbf{s}_{k} \phi^{\dagger} \partial_{j} \phi-i \mathbf{s} \times \partial_{i} \mathbf{s}\right] .
$$

Since $\mathbf{s}$ is a unit vector, Eq. (A3) follows.

We now move to the evaluation of the matrix elements $H_{1}$ and $H_{2}$. It can be proven that the term $H_{1}$ is the sum of the following terms:

$$
\begin{aligned}
I_{0} \equiv & \frac{1}{n} \operatorname{Re}\left\{\psi^{\dagger} \frac{(\hat{\mathbf{p}}-q \mathbf{A})^{2}}{2 m} \psi\right\}=\frac{\hbar^{2}}{2 m} \frac{\nabla^{2} \sqrt{n}}{\sqrt{n}}-\frac{\hbar^{2}}{8 m}\left(\partial_{i} s_{j}\right)^{2} \\
& -\frac{1}{2 m}\left(\partial_{i} S-q A_{i}-i \hbar \phi \partial_{i} \phi\right)^{2}, \\
I \equiv & \frac{1}{n} \operatorname{Re}\left\{\psi^{\dagger}\left[q V-\mu_{B} \boldsymbol{\sigma} \cdot \mathbf{B}-\frac{\hbar^{2} q}{8 m^{2} c^{2}} \nabla \cdot \mathbf{E}\right] \psi\right\} \\
= & q\left(V-\frac{\hbar^{2}}{8 m^{2} c^{2}} \nabla \cdot \mathbf{E}\right)-\mu_{B} \mathbf{s} \cdot \mathbf{B}, \\
I I \equiv & \frac{1}{n} \operatorname{Re}\left\{-\psi^{\dagger} \frac{\hbar q}{4 m^{2} c^{2}} \boldsymbol{\sigma} \cdot \mathbf{E} \times(\hat{\mathbf{p}}-q \mathbf{A}) \psi\right\}, \\
= & \frac{q \hbar}{4 m^{2} c^{2}} \mathbf{s} \cdot[\mathbf{E} \times(\nabla S-q \mathbf{A})]+\frac{q \hbar^{2}}{4 m^{2} c^{2}}(\mathbf{s} \times \mathbf{E}) \\
& \cdot \operatorname{Im}\left(\phi^{\dagger} \nabla \phi\right)+\frac{q \hbar^{2}}{8 m^{2} c^{2}} \epsilon_{i j k} E_{j} \epsilon_{i r l} s_{r} \partial_{k} s_{l}, \\
I I I \equiv & \frac{1}{n} \operatorname{Re}\left\{-\psi^{\dagger} \frac{i \hbar^{2} q}{8 m^{2} c^{2}} \boldsymbol{\sigma} \cdot \nabla \times \mathbf{E} \psi\right\}=0 .
\end{aligned}
$$

In particular, concerning the $I I$ matrix element, we used

$$
\begin{aligned}
I I= & \frac{q \hbar}{4 m^{2} c^{2}} \mathbf{s} \cdot[\mathbf{E} \times(\nabla S-q \mathbf{A})] \\
& +\frac{q \hbar^{2}}{4 m^{2} c^{2}} \mathbf{E} \cdot \operatorname{Im}\left(\phi^{\dagger} \boldsymbol{\sigma} \times \nabla \phi\right) .
\end{aligned}
$$

Furthermore, Eq. (A3) gives

$$
\begin{aligned}
\operatorname{Im}\left(\phi^{\dagger} \sigma_{i} \partial_{k} \phi\right) & =\frac{1}{2 i}\left\{s_{i}\left[\phi^{\dagger} \partial_{k} \phi-\left(\partial_{k} \phi^{\dagger}\right) \phi\right]\right\}+\frac{1}{2}\left(-\epsilon_{i r l} s_{r} \partial_{k} s_{l}\right) \\
& =-s_{i} \operatorname{Im}\left(\phi^{\dagger} \partial_{k} \phi\right)-\frac{1}{2} \epsilon_{i r l} s_{r} \partial_{k} s_{l}
\end{aligned}
$$

and we obtain Eq. (A13).

In the same way, we calculate the term $H_{2}$ as the sum of the following terms:

$$
\begin{aligned}
I_{0} & =\frac{1}{n} \operatorname{Im}\left\{\psi^{\dagger} \boldsymbol{\sigma} \frac{(\hat{\mathbf{p}}-q \mathbf{A})^{2}}{2 m} \psi\right\} \\
& =\frac{\hbar \mathbf{s}}{2 n}(\mathbf{v} \cdot \nabla) n+\frac{\hbar^{2}}{4 m} \partial_{j}\left(n \mathbf{s} \times \partial_{j} \mathbf{s}\right),
\end{aligned}
$$

$$
\begin{aligned}
I & =-\frac{1}{n} \operatorname{Im}\left\{\psi^{\dagger} \boldsymbol{\sigma}\left[q V-\mu_{B} \boldsymbol{\sigma} \cdot \mathbf{B}-\frac{\hbar^{2} q}{8 m^{2} c^{2}} \nabla \cdot \mathbf{E}\right] \psi\right\} \\
& =\mu_{B} \mathbf{S} \times \mathbf{B}, \\
I I & =\frac{\hbar q}{4 m^{2} c^{2}} \frac{1}{n} \operatorname{Im}\left\{\psi^{\dagger} \boldsymbol{\sigma}[\boldsymbol{\sigma} \cdot \mathbf{E} \times(i \hbar \nabla-q \mathbf{A}) \psi]\right\}, \\
& =-\frac{q \hbar}{4 m^{2} c^{2}}\left\{\frac{\hbar}{n}(\mathbf{E} \times \nabla n) \times \mathbf{s}+2 m(\mathbf{E} \times \mathbf{v})+\hbar \mathbf{a}\right\},
\end{aligned}
$$

$$
I I I=\frac{\hbar^{2} q}{8 m^{2} c^{2}} \frac{1}{n} \operatorname{Im}\left\{i \psi^{\dagger} \boldsymbol{\sigma}[\boldsymbol{\sigma} \cdot(\nabla \times \mathbf{E})] \psi\right\}=\frac{\hbar^{2} q}{4 m^{2} c^{2}} \nabla \times \mathbf{E},
$$

where we defined $a_{i} \equiv \epsilon_{i r l} \epsilon_{r j k} E_{j} \partial_{k} s_{l}$.

\section{APPENDIX B: MANY-PARTICLE AVERAGING}

Equations (6), (9), and (20) constitute the fluid equations in the single-particle approximation. The simplest way to describe an $\mathrm{N}$-particle system is to assume that the state of the system is represented by a statistical mixture of single-particle wave functions: $\psi_{\alpha}=\sqrt{n_{\alpha}} e^{i S_{\alpha} / \hbar} \phi_{\alpha}$, with a certain probability $p_{\alpha}(\alpha=1 \ldots N)$. In this framework, the expectation value of any quantity $A$ is obtained by the simple average

$$
\langle A\rangle \equiv \frac{1}{n} \sum_{\alpha} p_{\alpha} \psi_{\alpha}^{\dagger} A \psi_{\alpha},
$$

where $\langle n\rangle \equiv \sum_{\alpha} n_{\alpha}$ is the total density. Taking $A$ equal to $\mathbf{s}$ or $\mathbf{u}$ in Eq. (B1), we obtain the average spin density $\langle\mathbf{s}\rangle$ and average velocity $\langle\mathbf{u}\rangle$ :

$$
\begin{aligned}
\langle\mathbf{u}\rangle & =\frac{1}{n} \sum_{\alpha} p_{\alpha} n_{\alpha} \mathbf{u}_{\alpha}, \\
\langle\mathbf{s}\rangle & =\frac{1}{n} \sum_{\alpha} p_{\alpha} n_{\alpha} \mathbf{s}_{\alpha} .
\end{aligned}
$$

Applying the above averaging procedure to the microscopic continuity Eq. (6) (where we now add an index $\alpha$ to all the quantities) we obtain

$$
\partial_{t}\langle n\rangle+\nabla \cdot(\langle n\rangle\langle\mathbf{u}\rangle)=0 .
$$

From the statistical average of Eq. (24) we obtain

$$
\begin{aligned}
\frac{D\left\langle s_{i}\right\rangle}{D t}= & \mathcal{S}_{i}(\langle n\rangle,\langle\mathbf{s}\rangle,\langle\mathbf{u}\rangle)-\frac{1}{\langle n\rangle} \partial_{j} T_{i j} \\
& -\frac{q}{2 c^{2} m\langle n\rangle} \epsilon_{i j k} \epsilon_{k l m} T_{j l} E_{m},
\end{aligned}
$$

where we have defined the spin-velocity tensor

$$
T_{i j}=\langle n\rangle\left[\left\langle s_{i} u_{j}\right\rangle-\left\langle s_{i}\right\rangle\left\langle u_{j}\right\rangle\right] .
$$

The last two terms in Eq. (B5) constitute the first manybody correction to the single-particle equation for the spin density. The tensor $T_{i j}$ was also found in Ref. [39], where 
the nonrelativistic QHD model with spin was derived. The spin-velocity tensor can be interpreted as representing the correlations between the microscopic spins and velocities, just like the ordinary pressure tensor (see below) represents the correlations between the microscopic velocities. This tensor also appears naturally in the fluid equations derived from a kinetic theory [52] through a moment expansion. Note, however, that the spin-velocity tensor vanishes at thermal equilibrium [52].

In the derivation of Eq. (B5), we have also used the following approximations:

$$
\begin{gathered}
\sum_{\alpha} p_{\alpha} \frac{\hbar}{2 m} \epsilon_{i k l} \partial_{j}\left(n_{\alpha} s_{k, \alpha} \partial_{j} s_{l, \alpha}\right) \approx \frac{\hbar}{2 m} \epsilon_{i k l} \partial_{j}\left(\langle n\rangle\left\langle s_{k}\right\rangle \partial_{j}\left\langle s_{l}\right\rangle\right), \\
\sum_{\alpha} p_{\alpha} \frac{q \hbar}{4 m^{2} c^{2}} s_{i, \alpha} \mathbf{s}_{\alpha} \cdot \nabla \times\left(n_{\alpha} \mathbf{E}\right) \approx \frac{q \hbar}{4 m^{2} c^{2}}\left\langle s_{i}\right\rangle\langle\mathbf{s}\rangle \cdot \nabla \times(\langle n\rangle \mathbf{E}), \\
\sum_{\alpha} p_{\alpha} \frac{q \hbar}{2 m^{2} c^{2}} n_{\alpha}\left(\mathbf{E} \times \mathbf{s}_{\alpha}\right) \cdot \nabla s_{i, \alpha} \approx \frac{q \hbar}{2 m^{2} c^{2}}\langle n\rangle(\mathbf{E} \times\langle\mathbf{s}\rangle) \cdot \nabla\left\langle s_{i}\right\rangle .
\end{gathered}
$$

These approximations are all in the same spirit as the approximation that is usually employed for the Bohm potential, i.e.,

$$
\sum_{\alpha} p_{\alpha}\left(\frac{\partial_{x}^{2} \sqrt{n_{\alpha}}}{\sqrt{n_{\alpha}}}\right) \approx \frac{\partial_{x}^{2} \sqrt{\langle n\rangle}}{\sqrt{\langle n\rangle}}
$$

which was also used in the derivation of Eq. (B5) and is generally valid in the long wavelength limit [53]. Besides, we note that all terms in Eqs. (B7) are quadratic in the spin variable, and therefore will not change the linear response and the dispersion relations, although they may affect the nonlinear behavior of the system.

For the velocity field, the many particle averaging yields

$$
\begin{aligned}
\frac{D u_{i}}{D t}= & \mathcal{U}(\langle n\rangle,\langle\mathbf{s}\rangle,\langle\mathbf{u}\rangle)-\frac{1}{m\langle n\rangle} \partial_{j} P_{i j} \\
& -\frac{q \hbar}{4 m^{2} c^{2}\langle n\rangle}\left(\epsilon_{f j k} T_{j i} \partial_{i} E_{k}+\epsilon_{j k l} T_{j k} \partial_{f} E_{l}\right) \\
& -\frac{q \hbar}{4 m^{2} c^{2}} \epsilon_{j k l} W_{j f k} E_{l}-\frac{q \hbar}{4 m^{2} c^{2}} \epsilon_{i j k} E_{k} W_{j i f},
\end{aligned}
$$

where we have defined the pressure $P_{i j}$ and the spin-pressure $W_{i j k}$ tensors as follows:

$$
\begin{aligned}
P_{i j} & \equiv\langle n\rangle m\left[\left\langle u_{i} u_{j}\right\rangle-\left\langle u_{i}\right\rangle\left\langle u_{j}\right\rangle\right], \\
W_{i j k} & \equiv\langle n\rangle\left[\left\langle s_{i} \partial_{j} u_{k}\right\rangle-\left\langle s_{i}\right\rangle\left\langle\partial_{j} u_{k}\right\rangle\right] .
\end{aligned}
$$

In order to solve the many-body Eqs. (B5)-(B9), we need a suitable closure expression for the tensors $T_{i j}, P_{i j}$, and $W_{i j k}$. For the pressure tensor, a number of different choices can be made. In the isotropic case, we may write

$$
\frac{P_{i j}}{P_{0}}=\left(\frac{\langle n\rangle}{n_{0}}\right)^{\gamma} \delta_{i j},
$$

where $P_{0}$ and $n_{0}$ are reference values for the pressure and density. Depending on whether the plasma is degenerate or not, different values of $\gamma$ are required in order to agree with the results from kinetic theory. For instance, the choice $\gamma=3$ yields the correct dispersion relation for linear waves in a zero-temperature fully degenerate plasma [53].

It is more difficult to establish appropriate closure relations for the tensors involving the spin variables, $T$ and $W$. This important issue will be investigated in a forthcoming work.
[1] S. V. Bulanov, T. Z. Esirkepov, M. Kando, S. S. Bulanov, S. G. Rykovanov, and F. Pegoraro, Phys. Plasmas 20, 123114 (2013).

[2] S. H. Glenzer and L. Livermore, Rev. Mod. Phys. 81, 1625 (2009).

[3] Y. I. Salamin, S. X. Huc, K. Z. Hatsagortsyan, and C. H. Keitel, Phys. Rep. 427, 41 (2006).

[4] G. Mourou and T. Tajima, Science 331, 41 (2011).

[5] S. H. Glenzer et al., Phys. Rev. Lett. 98, 065002 (2007).

[6] E. Beaurepaire, J.-C. Merle, A. Daunois, and J.-Y. Bigot, Phys. Rev. Lett. 76, 4250 (1996).

[7] J.-Y. Bigot, M. Vomir, and E. Beaurepaire, Nat. Phys. 5, 515 (2009).

[8] J.-Y. Bigot and M. Vomir, Ann. Phys. (Berlin) 525, 2 (2013).

[9] J.-Y. Bigot, Nature Mater. 12, 283 (2013).

[10] B. Vodungbo et al., Nature Commun. 3, 999 (2012).

[11] T. Wang et al., Phys. Rev. Lett. 108, 267403 (2012)

[12] D. Kiefer et al., Nature Commun. 4, 1763 (2013).

[13] C. Joshi and T. Katsouleas, Phys. Today 56, 47 (2003).

[14] B. Dromey et al., Nature Phys. 8, 804 (2012).

[15] M. M. Seibert et al., Nature 470, 78 (2011).

[16] S. White et al., High Energy Dens. Phys. 9, 573 (2013).

[17] R. Neutze, R. Wouts, D. van der Spoel, E. Weckert, and J. Hajdu, Nature 406, 752 (2000).
[18] C. Boeglin, O. Ersen, M. Pilard, V. Speisser, and F. Kronast, Phys. Rev. B 80, 035409 (2009).

[19] A. Pukhov, S. Gordienko, V. Seredov, and I. Kostyukov, C. R. Phys. (Paris) 10, 159 (2009).

[20] J. Meyer-ter-Vehn and H.-C. Wu, Eur. Phys. J. D 55, 433 (2009).

[21] B. Qiao, M. Zepf, M. Borghesi, B. Dromey, and M. Geissler, New J. Phys. 11, 103042 (2009).

[22] M. W. Walser and C. H. Keitel, J. Phys. B: Atom. Mol. Opt. Phys. 33, L221 (2000).

[23] J. Zhu and P. Ji, Phys. Rev. E 81, 036406 (2010).

[24] G. R. Mocken and C. H. Keitel, Phys. Rev. Lett. 91, 173202 (2003).

[25] Julio San Roman, Luis Roso, and Luis Plaja, J. Phys. B: Atom. Mol. Opt. Phys. 37, 435 (2004).

[26] Kenneth G. Dyall and Knut Fægri Jr., Introduction to Relativistic Quantum Chemistry (Oxford University Press, Oxford, 2007).

[27] E. Engel, R. M. Dreizler, S. Varga, and B. Fricke, in Relativistic Effects in Heavy-Element Chemistry and Physics, edited by B. A. Hess (Wiley, New York, 2003).

[28] A. I. Rajagopal, J. Phys. C: Solid State Phys. 11, L943 (1978).

[29] F. A. Parpia and W. R. Johnson, J. Phys. B: Atom. Mol. Phys. 17, 531 (1984). 
[30] P. Romaniello and P. L. de Boeij, J. Chem. Phys. 127, 174111 (2007).

[31] L. L. Foldy and S. A. Wouthuysen, Phys. Rev. 78, 29 (1950).

[32] J. D. Bjorken and S. D. Drell, Relativistic Quantum Mechanics (McGraw-Hill Book Company, New York, 1964).

[33] Y. Hinschberger and P.-A. Hervieux, Phys. Lett. A 376, 813 (2012).

[34] E. van Lenthe, E. J. Baerends, and J. G. Snijders, J. Chem. Phys. 99, 4597 (1993).

[35] A. Dixit, Y. Hinschberger, J. Zamanian, G. Manfredi, and P.-A. Hervieux, Phys. Rev. A 88, 032117 (2013).

[36] E. Madelung, Zeitschr. Phys. 40, 322 (1926).

[37] D. Bohm, Phys. Rev. 85, 166 (1952).

[38] G. Manfredi and F. Haas, Phys. Rev. B 64, 075316 (2001).

[39] G. Brodin and M. Marklund, New. J. Phys 9, 277 (2007).

[40] P. R. Holland, The Quantum Theory of Motion (Cambridge University Press, Cambridge, 1993).

[41] M. Stefan and G. Brodin, Phys. Plasmas 20, 012114 (2013).

[42] F. Asenjo, J. Zamanian, M. Marklund, G. Brodin, and P. Johansson, New J. Phys. 14, 073042 (2012).
[43] F. Asenjo, V. Muñoz, J. A. Valdivia, and S. M. Mahajan, Phys. Plasmas 18, 012107 (2011).

[44] T. Takabayasi, Prog. Theor. Phys. Suppl. 4, 2 (1957).

[45] L. D. Landau and E. M. Lifshitz, Quantum Mechanics: NonRelativistic Theory (Butterworth-Heinemann, Oxford, 1977).

[46] T. Jungwirth, J. Sinova, J. Masek, J. Kuèera, and A. H. MacDonald, Rev. Mod. Phys. 78, 809 (2006).

[47] I. Zutic, J. Fabian, S. Das Sarma, Rev. Mod. Phys. 76, 323 (2004).

[48] G. Manfredi and P.-A. Hervieux, Phys. Rev. B 72, 155421 (2005).

[49] R. Jasiak, G. Manfredi, P.-A. Hervieux, and M. Haefele, New J. Phys. 11, 063042 (2009).

[50] M. G. Haines, M. S. Wei, F. N. Beg, and R. B. Stephens, Phys. Rev. Lett. 102, 045008 (2009).

[51] C. P. Ridgers, C. S. Brady, R. Duclous, J. G. Kirk, K. Bennett, T. D. Arber, and A. R. Bell, Phys. Plasmas 20, 056701 (2013).

[52] J. Zamanian, M. Stefan, M. Marklund, and G. Brodin, Phys. Plasmas 17, 102109 (2010).

[53] G. Manfredi, in Topics in Kinetic Theory, edited by T. Passot, C. Sulem, and P.-L. Sulem (American Mathematical Society, Toronto, 2005). 